\title{
COMMENTS ON CANONICAL LINES
}

\author{
R. B. RASMUSEN AND B. L. HAGEN
}

1. Introduction. In this paper we propose to find the equations of the canonical edges of Green using a conjugate net as the parametric net of an analytic surface; to give a new interpretation to the line called by Davis the associate line of collineation; and, finally, to make a generalization of the canonical quadric of Davis.

2. Analytic basis. Let the projective homogeneous coordinates $x^{1}, \cdots, x^{4}$ of a point $P_{x}$ on a surface $S$ in ordinary space be analytic functions of two independent variables $u, v$. In the notation ${ }^{1}$ of Lane if the parametric curves on $S$ form a conjugate net, the coordinates $x$ of the point $P_{x}$ and the coordinates $y$ of a point which is the harmonic conjugate of the point $P_{x}$ with respect to the foci of the axis of the point $P_{x}$, satisfy a system of equations of the form

$$
\begin{aligned}
& x_{u u}=p x+\alpha x_{u}+L y, \\
& x_{u v}=c x+a x_{u}+b x_{v}, \\
& x_{v v}=q x+\delta x_{v}+N y, \quad L N \neq 0 .
\end{aligned}
$$

The ray-points of the net at the point $P_{x}$ are given by the formulas

$$
x_{-1}=x_{u}-b x, \quad x_{1}=x_{v}-a x .
$$

Some of the invariants of the net are

$$
\begin{aligned}
H & =c+a b-a_{u}, & K & =c+a b-b_{v}, \\
\mathfrak{S} & =c+a b+b_{v}-\delta_{u}, & \Re & =c+a b+a_{u}-\alpha_{v}, \\
8 \mathfrak{B}^{\prime} & =4 a-2 \delta+(\log r)_{v}, & r & =N / L, \\
8 \mathfrak{S}^{\prime} & =4 b-2 \alpha-(\log r)_{u} . & &
\end{aligned}
$$

If the covariant tetrahedron, $x, x_{1}, x_{-1}, y$ is used as the local tetrahedron of reference, a power series expansion ${ }^{2}$ for one nonhomogeneous coordinate $z$ of a point on the surface in terms of the other two coordinates $x, y$ is

$$
\begin{aligned}
z= & \frac{1}{2}\left(L x^{2}+N y^{2}\right)+\frac{4}{3}\left(L \mathscr{C}^{\prime} x^{3}+N \mathscr{B} y^{3}\right)+c_{0} x^{4} \\
& +4 c_{1} x^{3} y+4 c_{3} x y^{3}+c_{4} y^{4}+\cdots
\end{aligned}
$$

\footnotetext{
${ }^{1}$ Lane, Conjugate nets and the lines of curvature, American Journal of Mathematics, vol. 53 (1931), p. 574.

${ }^{2}$ Lane, A canonical power series expansion for a surface, Transactions of this Society, vol. 37 (1935), p. 481.
} 
where the coefficients $c_{0}, c_{1}, c_{3}, c_{4}$ are given by

$$
\begin{array}{ll}
c_{0}=\frac{1}{3} L \mathfrak{S}^{\prime}\left[12 \mathfrak{S}^{\prime}+\left(\log \left(\mathfrak{S}^{\prime} r^{1 / 2}\right)_{u}\right],\right. & 4 c_{1}=\frac{1}{6} L(H-\mathfrak{S}), \\
c_{4}=\frac{1}{3} N \mathfrak{B}^{\prime}\left[12 \mathfrak{B}^{\prime}+\left(\log \mathfrak{B}^{\prime} r^{-1 / 2}\right)_{v}\right], & 4 c_{3}=\frac{1}{6} N(K-\mathfrak{\Re}),
\end{array}
$$

and the coefficient of $x^{2} y^{2}$ is zero.

We will assume that the surface $S$ is non-ruled, ${ }^{3}$ that is, that $\mathfrak{B}^{\prime 2}+r \mathfrak{S}^{\prime 2} \neq 0$.

3. The second canonical edge of Green. Segre has shown ${ }^{4}$ that the second $^{5}$ edge of Green is the line of inflexion of the nodal cubic in which the tangent plane at a point $P_{x}$ of an analytic surface $S$ intersects the four-parameter family of nondegenerate algebraic cubic surfaces having fourth order contact with $S$ at $P_{x}$.

The equation in nonhomogeneous coordinates of a general algebraic cubic surface through the point $P_{x}$ is

$$
\begin{aligned}
a_{1} x+a_{2} y & +a_{3} z+b_{1} x^{2}+b_{2} y^{2}+b_{3} z^{2}+b_{4} x y+b_{5} x z \\
& +b_{6} y z+d_{1} x^{3}+d_{2} y^{3}+d_{3} z^{3}+d_{4} x^{2} y+d_{5} x^{2} z+d_{6} x y^{2} \\
& +d_{7} y^{2} z+d_{8} x z^{2}+d_{9} y z^{2}+d_{10} x y z=0 .
\end{aligned}
$$

If this surface has fourth order contact with the surface $S$ at the point $P_{x}$, its coefficients satisfy the following conditions:

$$
\begin{aligned}
& a_{1}=a_{2}=b_{4}=0, \quad a_{3} L+2 b_{1}=0, \quad a_{3} N+2 b_{2}=0, \\
& 8 L \mathfrak{S}^{\prime} a_{3}+3 L b_{5}+d_{1}=0, \quad L b_{6}+2 d_{4}=0, \\
& 8 N \mathfrak{B}^{\prime} a_{3}+3 N b_{6}+d_{2}=0, \quad N b_{5}+2 d_{6}=0, \\
& 12 c_{0} a_{3}+3 L^{2} b_{3}+16 L \mathfrak{S}^{\prime} b_{5}+6 L d_{5}=0, \\
& 12 c_{4} a_{3}+3 N^{2} b_{3}+16 N \mathfrak{B}^{\prime} b_{6}+6 N d_{7}=0, \\
& 12 c_{1} a_{3}+8 L \mathscr{S}^{\prime} b_{6}+3 L d_{10}=0, \\
& 12 c_{3} a_{3}+8 N \mathfrak{B}^{\prime} b_{5}+3 N d_{10}=0, \\
& L N b_{3}+N d_{5}+L d_{7}=0 .
\end{aligned}
$$

The tangent plane, $z=0$, intersects this cubic surface in the cubic curve

${ }^{3}$ Green, Projective differential geometry of one-parameter families of curves and conjugate nets on a curved surface, First Memoir, American Journal of Mathematics, vol. 37 (1915), p. 239.

${ }^{4} \mathrm{~B}$. Segre, La cubique indicatrice de l'êlément linéaire projectif d'une surface, Comptes Rendus de l'Académie des Sciences, Paris, vol. 184, p. 729.

${ }^{5}$ This classification of the edges of Green is the reverse of that introduced by Green. 


$$
z=b_{1} x^{2}+b_{2} y^{2}+d_{1} x^{3}+d_{2} y^{3}+d_{4} x^{2} y+d_{6} x y^{2}=0,
$$

where the coefficients $b_{1}, b_{2}, d_{1}, d_{2}, d_{4}, d_{6}$ can be expressed in terms of the invariants given by equations (2) and $a_{3}$.

Following the usual method of solving a cubic with its Hessian and discarding the solution giving the double point tangents, we obtain the following equation of the line of inflexions of the cubic:

$$
z=a_{3}+\left(b_{5}+2 \mathfrak{S}^{\prime} a_{3}\right) x+\left(b_{6}+2 \mathfrak{B}^{\prime} a_{3}\right) y=0,
$$

where

$$
\begin{aligned}
b_{5}= & \frac{-a_{3}}{8\left(N \mathfrak{C}^{\prime 2}+L \mathfrak{B}^{\prime 2}\right)}\left\{-L \mathfrak{B}^{\prime}[(H-\mathfrak{S})-(K-\mathfrak{R})]\right. \\
& +2 \mathfrak{S}^{\prime}\left[12\left(N \mathfrak{S}^{\prime 2}+L \mathfrak{B}^{\prime 2}\right)+N \mathfrak{S}^{\prime}\left(\log \mathfrak{S}^{\prime} \boldsymbol{r}^{1 / 2}\right)_{u}\right. \\
& \left.\left.+L \mathfrak{B}^{\prime}\left(\log \mathfrak{B}^{\prime} r^{-1 / 2}\right)_{v}\right]\right\},
\end{aligned}
$$

$$
\begin{aligned}
b_{6}= & \frac{-a_{3}}{8\left(N \mathfrak{S}^{\prime 2}+L \mathfrak{B}^{\prime 2}\right)}\left\{N \mathfrak{S}^{\prime}[(H-\mathfrak{S})-(K-\Re)]\right. \\
& +2 \mathfrak{B}^{\prime}\left[12\left(N \mathfrak{S}^{\prime 2}+L \mathfrak{B}^{\prime 2}\right)+N \mathfrak{S}^{\prime}\left(\log \left(\mathfrak{S}^{\prime} \boldsymbol{r}^{1 / 2}\right)_{u}\right.\right. \\
& \left.\left.+L \mathfrak{B}^{\prime}\left(\log \mathfrak{B}^{\prime} r^{-1 / 2}\right)_{v}\right]\right\} .
\end{aligned}
$$

Any line of the second canonical pencil ${ }^{6}$ of Davis may have its equation in the form

$$
z=1+k\left(\complement^{\prime} x+\mathfrak{B}^{\prime} y\right)=0,
$$

where $k$ is a constant. The flex-ray (7) is a line of this pencil in case

$$
2 \mathfrak{S}^{\prime}+b_{5} / a_{3}=k \mathbb{S}^{\prime}, \quad 2 \mathfrak{B}^{\prime}+b_{6} / a_{3}=k \mathfrak{B}^{\prime} .
$$

Eliminating $k$ from these equations, we obtain

$$
\left(\mathfrak{B}^{\prime 2}+r \mathfrak{C}^{\prime 2}\right)[(H-\mathfrak{S})-(K-\mathfrak{\Omega})]=0 .
$$

Therefore, necessary and sufficient conditions for the second edge of Green to be a line of the second canonical pencil of Davis are

$$
\begin{gathered}
H-\mathfrak{S}=K-\Re, \\
{\left[r \mathfrak{S}^{\prime}\left(\log \left(\mathfrak{S}^{\prime} r^{1 / 2}\right)_{u}+\mathfrak{B}^{\prime}\left(\log \mathfrak{B}^{\prime} r^{-1 / 2}\right)_{v}\right] /\left(\mathfrak{B}^{\prime 2}+r \mathfrak{C}^{\prime 2}\right)=\right.\text { const. }}
\end{gathered}
$$

A line of the first canonical pencil ${ }^{7}$ of Davis joins the points $(1,0,0,0)$ and $\left(0,-k \mathfrak{S}^{\prime} / L,-k \mathfrak{B}^{\prime} / N, 1\right)$. It can be verified that the

${ }^{6}$ W. M. Davis, Contributions to the Theory of Conjugate Nets, Chicago Doctor's Thesis, 1933, p. 18.

${ }^{7}$ Davis, ibid., p. 20. 
first edge of Green is the line joining the point $(1,0,0,0)$ to the point $\left[0,-\left(b_{5} / a_{3}+4 \mathfrak{E}^{\prime}\right) / L,-\left(b_{6} / a_{3}+4 \mathfrak{B}^{\prime}\right) / N, 1\right]$. The first edge of Green is a line of the first canonical pencil of Davis if conditions (9) hold. This is a special case of the following theorem. ${ }^{8}$

If one line of an arbitrarily selected pair of lines which are reciprocal with respect to a quadric of Darboux is a line of the second canonical pencil of Davis, its reciprocal is a line of the first canonical pencil of Davis, and conversely.

4. The associate line of collineation. The cubic surface

$$
z=\frac{1}{2}\left(L x^{2}+N y^{2}\right)+\frac{4}{3}\left(L \mathbb{S}^{\prime} x^{3}+N \mathfrak{B}^{\prime} y^{3}\right)
$$

obtained by truncating the power series (3) can be interpreted ${ }^{9}$ geometrically as follows: first, it has third order contact with the analytic surface $S$ given by equations (3) at the point $(1,0,0,0)$; second, it has a unode of second order at the point $(0,0,0,1)$. This cubic surface intersects the tangent plane $z=0$ in the nodal cubic

$$
z=3\left(x^{2}+r y^{2}\right)+8\left(\mathbb{C}^{\prime} x^{3}+r \mathfrak{B}^{\prime} y^{3}\right)=0
$$

which has for its line of inflexion $z=1+2\left(\mathfrak{S}^{\prime} x+\mathfrak{B}^{\prime} y\right)=0$. This is the associate line of collineation, ${ }^{10}$ and is a line of the second canonical pencil of Davis.

5. A generalization of the canonical quadric of Davis. Davis has shown ${ }^{11}$ that at each point $P_{x}$ of a conjugate net on an analytic surface $S$ there exists a unique quadric surface which has the following properties: the quadric has second order contact with the surface sustaining the net; the axis and ray of the net at $P_{x}$ are reciprocal polars with respect to the quadric; and the quadric passes through the point $P_{y}$ which is the harmonic conjugate of $P_{x}$ with respect to the focal points of the axis. We shall generalize this definition by showing that at each point $P_{x}$ of a conjugate net there exists a two-parameter family of quadric surfaces having the following properties: the quadrics have second order contact with the surface sustaining the net and any line of the second canonical pencil of Davis has as its polar reciprocal with respect to any quadric of this family the line of the first canonical pencil defined by the same value of $k$.

${ }^{8}$ We are indebted to P. O. Bell for this theorem in its general form.

${ }^{\circ}$ Lane, Projective Differential Geometry of Curves and Surfaces, The University of Chicago Press, 1932, p. 296.

${ }^{10}$ Davis, ibid., p. 25.

${ }^{11}$ Davis, ibid., p. 11. 
The equation ${ }^{12}$ of all quadric surfaces having second order contact with the analytic surface $S$, given by equation (3), is

$$
L x^{2}+N y^{2}+z\left(-2+k_{2} x+k_{3} y+k_{4} z\right)=0,
$$

where $k_{2}, k_{3}, k_{4}$ are arbitrary constants when $P_{x}$ is fixed, and functions of $u, v$ when $P_{x}$ varies over $S$.

If we demand that the polar reciprocals of any line of the first canonical pencil of Davis with respect to a quadric given by equation (10) be the line of the second canonical pencil of David defined by the same value of $k$, we obtain $k_{2}=-4 k \mathfrak{S}^{\prime}, k_{3}=-4 k \mathfrak{B}^{\prime}$. Substituting these values in (10), we have

$$
L x^{2}+N y^{2}+z\left(-2-4 k \mathbb{S}^{\prime} x-4 k \mathfrak{B}^{\prime} y+k_{4} z\right)=0 .
$$

If $k=0$, and the quadric passes through the point $P_{y}$ we have the canonical quadric of Davis; also if $k=-1$, we have the quadrics of Darboux.

It is interesting to note that for $k=-1$ the line in the second canonical pencil of Davis is the line of inflexions of the ray-point cubic. ${ }^{13}$

The second canonical tangent ${ }^{14}$ of Davis is the line joining the canonical point $\left(0, \mathfrak{B}^{\prime},-\mathfrak{S}^{\prime}, 0\right)$ to the point $P(1,0,0,0)$. The coordinates of any point on this line are

$$
\left(\lambda, \mu \mathfrak{B}^{\prime},-\mu\left(\overleftarrow{S}^{\prime}, 0\right),\right.
$$

where $\lambda$ and $\mu$ are arbitrary constants. The polar plane of this point with respect to the quadrics whose equation is given by (11) is

$$
\mu\left(L \mathfrak{B}^{\prime} x-N \mathfrak{S}^{\prime} y\right)-\lambda z=0,
$$

which is independent of $k$.

For $\lambda=0$, the point given by (12) is the canonical point of Davis and the plane (13) is the canonical plane of Davis.

Consequently, we have the theorem: the polar plane of a point on the second canonical tangent of Davis with respect to a quadric of the family defined by equation (11) is a member of a pencil whose axis is the first canonical tangent of Davis. This plane is dependent on the selection of the point but is independent of the selection of the quadric of the family (11).

Woodrow Wilson Junior College and

Pennsylvania State College

12 Davis, ibid., p. 11.

13 Lane, Conjugate nets and the lines of curvature, American Journal of Mathematics, vol. 53 (1931), p. 577.

${ }^{14}$ Davis, ibid., pp. 17-18. 